\title{
Localization of binary neutron star mergers with second and third generation gravitational-wave detectors
}

\author{
Cameron Mills, Vaibhav Tiwari, and Stephen Fairhurst \\ Cardiff School of Physics and Astronomy, Cardiff University, Queens Buildings, \\ The Parade, Cardiff CF24 3AA, United Kingdom
}

(Received 3 August 2017; published 29 May 2018)

\begin{abstract}
The observation of gravitational wave signals from binary black hole and binary neutron star mergers has established the field of gravitational wave astronomy. It is expected that future networks of gravitational wave detectors will possess great potential in probing various aspects of astronomy. An important consideration for successive improvement of current detectors or establishment on new sites is knowledge of the minimum number of detectors required to perform precision astronomy. We attempt to answer this question by assessing the ability of future detector networks to detect and localize binary neutron stars mergers on the sky. Good localization ability is crucial for many of the scientific goals of gravitational wave astronomy, such as electromagnetic follow-up, measuring the properties of compact binaries throughout cosmic history, and cosmology. We find that although two detectors at improved sensitivity are sufficient to get a substantial increase in the number of observed signals, at least three detectors of comparable sensitivity are required to localize majority of the signals, typically to within around $10 \mathrm{deg}^{2}$ —adequate for follow-up with most wide field of view optical telescopes.
\end{abstract}

DOI: 10.1103/PhysRevD.97.104064

\section{INTRODUCTION}

One hundred years after gravitational waves were predicted [1], the first detection of a binary black hole (BBH) coalescence by the advanced LIGO detectors [2] heralded the beginning of the era of gravitational wave (GW) astronomy. Less than two years later, and with the advanced Virgo detector also in operation, the detection of GWs from a binary neutron star (BNS) merger marked another landmark event [3]. Over the coming years, the sensitivity of the advanced LIGO and Virgo instruments will improve, and the KAGRA and LIGO India detectors will join the global network [4-8]. This network of advanced gravitational wave detectors is expected to observe many more $\mathrm{BBH}$ and BNS mergers, as well as GWs emitted during the merger of neutron star-black hole (NSBH) binaries $[9,10]$. Additionally, GWs emitted by nonsymmetric neutron stars, core-collapse supernovae, and other astrophysical transient events may be observed [11,12].

The observation of electromagnetic (EM) counterparts to GWs is a major goal for astronomy and will be critical for extracting the maximum science from future events. Despite the expectation that stellar mass $\mathrm{BBH}$ mergers do not produce electromagnetic signals, there was broadband follow-up of GW150914 [13]. This demonstrated the willingness of the wider astronomical community to engage in multimessenger observation of GW sources. In contrast to BBHs, compact binary systems composed of at least one neutron star have plausible EM counterparts across gamma, x-ray, optical, infrared, and radio bandwidths (for possible counterparts see [14-22]). Indeed, the follow-up campaign for the BNS merger GW170817 was phenomenally successful, measuring counterparts across the EM spectrum [23-46]. This multimessenger data provided convincing answers to many outstanding questions. For instance, the detection of a short gamma ray burst (GRB) 1.7 seconds after GW170817 [24-26], and subsequent kilonova [27-31,36-44], confirmed that BNS mergers are a progenitor of these events. Lanthanide signatures in the kilonova light curves also showed BNS mergers to be a major site for nucleosynthesis of elements heavier than iron $[40,44,47,48]$. Furthermore the measurements of the EM redshift and, from the GW signal, the luminosity distance, allowed an independent estimate of the Hubble constant to be made [49], thus demonstrating a thirty year old prediction [50]. Crucial to these scientific results was the localization provided by the LIGO and Virgo interferometers.

Our ability to measure counterparts, and statistically identify host galaxies when no counterparts are present, depends on the GW localization. Unlike most EM telescopes, GW detectors are not pointing instruments, and localization is achieved primarily by measuring the differences in arrival times of the signal in different detectors [51]. Consequently, searching the relatively large GW localization regions $\left(\mathcal{O}\left(100-1000 \mathrm{deg}^{2}\right)\right.$ for the first detections $[11,52,53])$ represents a challenge for even wide field of view UV, optical and infrared telescopes. These telescopes have fields of view on the order of $10 \mathrm{deg}^{2}$ or 
less [54-56]. The addition of Virgo has improved the localization ability of the network by about an order magnitude [3,57]. Extra detectors in India and Japan will further reduce localization regions, allowing many signals to be localized to within tens of square degrees [6,58-60].

There are plans for future gravitational wave detectors that will be significantly more sensitive than the current generation of advanced detectors. These include upgrades to the existing detectors, such as $A+$ and LIGO Voyager [61], which gives the best possible sensitivity within the current LIGO infrastructure. Additionally, entirely new detectors have been proposed. The Einstein Telescope is a next-generation European gravitational wave observatory [62-64], and Cosmic Explorer [65] is a proposed US-based future detector, both of which improve on the advanced detector sensitivity by a factor of 10 or more. As well as revealing new sources of gravitational waves, these detectors will allow us to observe BBH mergers throughout most of the history of the Universe [66] and BNS to cosmological distances [67-70]. Furthermore, the nearby signals will be very loud in these detectors, allowing for unprecedented tests of Einstein's general relativity and observation of matter at supranuclear density inside neutron stars. As with the advanced detector network, joint GW-EM observations will be vital in fully extracting the science from these observations $[15,19,71,72]$.

The science case for these new facilities is still evolving and will continue to do so as further gravitational wave observations are made. Estimates of the accuracy with which networks of third and second generation detectors can reconstruct parameters will inform decisions over the viability of new facilities. There have been previous studies of ET that estimate the detection efficiency and the accuracy of mass measurements [68-70,73]. Estimates of the localization ability of various third generation networks were also considered as part of a comprehensive parameter estimation study [66], and in analytical studies focusing on the low frequency benefits of $3 \mathrm{G}$ detectors [74] and the implications for cosmology [75]. Furthermore, detailed studies of the optimal location of future detectors have been performed [76-78].

One practical consideration is whether it would be advantageous to accelerate the development of third generation detectors, perhaps at the expense of further upgrades to the second generation, or if the operation of a heterogeneous network of detectors is preferable. To date, there is rather little in the literature on the merits of such networks. Here we investigate the differences between homogeneous and heterogeneous networks of detectors. For concreteness, we focus primarily on the sky coverage of the networks and the accuracy with which they are able to localize sources. We consider the network localization accuracy for both face-on BNS systems at a fixed distance as well as a population of BNS distributed isotropically and with a redshift distribution that follows the star formation rate shifted to account for the delay between star formation and binary merger.

Previous estimates of network localization errors largely fall into two distinct categories: the first being analytical estimates that bypass the full task of parameter estimation and reduce the parameter space by focusing primarily on source localization [51,58,77,79-88]; the second being full parameter estimation studies that extract detailed parameter estimates using Bayesian statistics [59,89-96]. Performing the full analysis has the advantage of being more accurate, but due to the computational cost the number of sources that can be considered is typically small. On the other hand, analytical studies using only the timing information [51,85] have been shown to overestimate the localization error region [97]. Here, we make use of an improved, analytical method that incorporates amplitude and phase consistency between the sites, as well as timing [98].

This paper is organized as follows. Section II will describe the networks used in this study. Section III introduces the method for calculating the localization error regions. We present and analyse our main results in Sec. IV before concluding in Sec. V.

\section{FUTURE DETECTORS AND NETWORKS}

\section{A. Future detectors}

GW detector sensitivity is limited by a number of fundamental noise sources. These noise sources can be broadly separated into two categories: displacement noise and sensing noise. Displacement noises cause motions of the test masses. Noise sources such as seismic noise and mechanical resonances are in this category. Sensing noises, on the other hand, are phenomena that limit the ability to measure those motions; they are present even in the absence of test mass motion. Shot noise and thermal noise are included in this category. In addition, there are technical noise sources which must be understood and mitigated in order that the detector sensitivity is limited by fundamental noise. Typically, low frequency sensitivity is limited by seismic noise, mid frequencies are limited by thermal noise and higher frequencies are limited by quantum noise. LIGO underwent a series of upgrades from its initial to advanced configuration to address each of the noise sources [99]. Seismic noise is being suppressed by the use of multistage mechanical seismic isolation and quadruple pendulum suspension systems. Thermal noise arises in test masses and suspensions and is determined by material properties and beam size. Compared to initial LIGO, advanced LIGO uses a larger beam size. This results in better averaging of beam on a larger surface area which combined with better coating and suspension material results in efficient dissipation of heat. Quantum noise arises due to statistical fluctuations in the detected photon arrival rate. Quantum noise is overcome by increasing the beam power and 
increasing the weight of the test masses to overcome the increased radiation pressure.

Many technologies have been proposed to further increase the sensitivity of ground based detectors. For example, building detectors underground to suppress gravity gradients [62], improving mirror coatings (Sec. 5.9.3 in [100]) and cryogenically cooling the mirrors for reducing the thermal noise, and using squeezed light for lowering the noise floor due to quantum noise [101]. A detailed discussion on possible technology improvements is given in [100]. In the following, we briefly introduce several proposed future detector configurations and their corresponding sensitivities (see Fig. 1). These are used in the following sections when comparing the performance of different networks.

LIGO Voyager: Various upgrades have been proposed for the advanced LIGO detectors [61] leading to the proposal for an upgrade to $A+$ in 2020 followed by a further upgrade to LIGO Voyager which is envisioned to be operational around 2025. Voyager improves on the sensitivity of advanced LIGO by around a factor of 3 across a broad frequency range. The increased sensitivity is intended to be achieved by improvements in all the departments (seismic isolation system, coatings of mirrors, heavier and larger test masses, increased beam power, etc.) of the advanced LIGO infrastructure combined with frequency dependent squeezing and cryogenic cooling of mirrors [102-104].

Einstein Telescope: Various studies have shown that further increase in sensitivity is required for performing precise gravitational wave astronomy, testing of general relativity and improving our understanding of exotic phenomenon like the equation of state and tidal deformability of neutron stars [14,67,105-107]. The Einstein Telescope is a proposed next-generation European gravitational wave observatory [62-64] with sensitivity an order of magnitude higher than advanced LIGO and extending down to $1 \mathrm{~Hz}$. It intends to achieve this improvement through a combination of longer arms and improved technologies. The original design called for a triangular configuration of three interferometers with $10 \mathrm{~km}$ arms and $60^{\circ}$ angle between the arms. In addition, the proposed xylophone configuration allows installation of separate high and low-frequency detectors. High frequency sensitivity is most easily achieved with high laser power, but this generates significant complications at lower frequencies. The divided detector avoids this issue by allowing us to pursue different strategies in optimizing the noise for each frequency range. Additionally, it also reduces the length of tunnel required (as each tunnel is used by two of the interferometers) and also makes the detector sensitive to both gravitational wave polarizations [100].

Cosmic Explorer: There is also a proposal for a Cosmic Explorer detector $[61,65]$, which would be around a factor of 3 more sensitive than ET. The design and technology used is similar to ET but with arm length that can stretch out

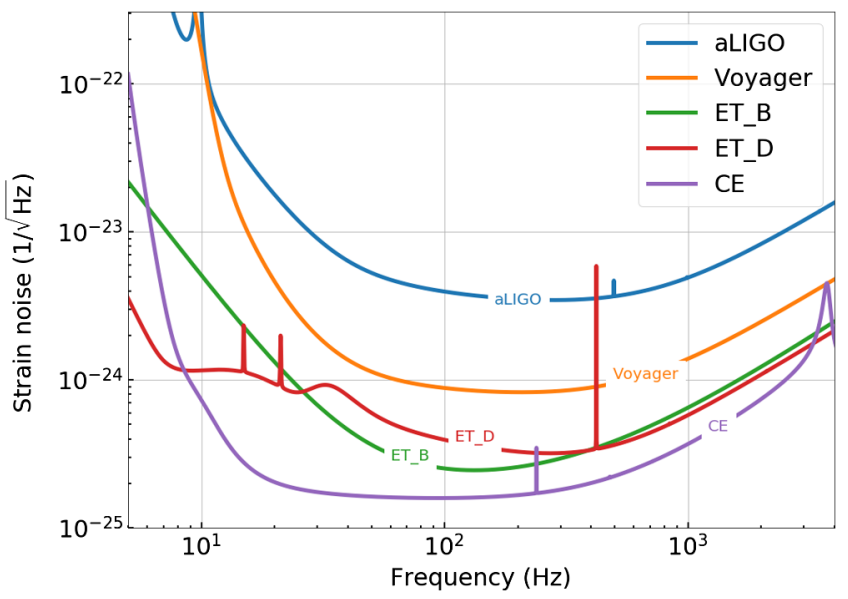

FIG. 1. Target noise curves for existing and future detectors [65]: advanced LIGO at design sensitivity (aLIGO); LIGO Voyager; Einstein Telescope (two proposed configurations, ET-B and ET-D) and Cosmic Explorer (CE).

to between 40 to $50 \mathrm{~km}$. Although the possibility of these detectors only lies in the far future, it is noteworthy that these detectors would see GW150914 like BBH mergers throughout the visible Universe.

In Fig. 1 we show the sensitivities of the proposed future detectors [65], as well as the advanced LIGO design sensitivity. We show the ET xylophone configuration, called ET-D. Also included for comparison is ET-B, which is an alternative ET configuration where every interferometer is optimized for best overall sensitivity, but at the expense of some low frequency sensitivity. For all ET simulations in this study, ET-D sensitivity is assumed.

\section{B. Networks}

We will consider five networks of gravitational wave observatories beyond the advanced detectors that are currently being built, commissioned and operated. Specifically, we consider:

(i) A network comprising detectors at the three LIGO sites (Hanford, Livingston and India) where the detectors have been upgraded to LIGO Voyager sensitivity. (Voyager)

(ii) A network comprising the three LIGO Voyager detectors complemented by a triangular ET detector in Europe. (Voyager-ET)

(iii) A network with three L-shaped detectors at ET sensitivity distributed globally. (3ET)

(iv) A network comprising a triangular ET detector and two Cosmic Explorer detectors (CE-ET)

(v) A network of three Cosmic Explorer detectors (3CE).

Networks (i) and (ii) arise naturally from existing proposals, but there is currently no global plan for a third generation network. Although there is no proposal for a network of ET detectors, we include this as configuration (iii), 
to facilitate comparison with the heterogeneous Voyager-ET network. It can be shown that the triangular ET detector has the same sensitivity to the two gravitational-wave polarizations as two colocated L-shaped detectors of length $10.6 \mathrm{~km}$ whose orientations differ by $45^{\circ}[62] .{ }^{1}$ For simplicity, we use a network of L-shaped ET detectors with three detectors each of comparable sensitivity to one of the two effective L-shaped detectors in the triangular ET. However, the freedom to orient and place the detectors far apart results in a network typically with improved localization and diminished ability to resolve both polarizations. We also consider a comparable network comprised of Cosmic Explorer detectors as well as a heterogeneous CE-ET network. Both the Voyager-ET and CE-ET networks exhibit substantial heterogeneity of sensitivities with a factor of 3 difference over a broad frequency range. The majority of previous studies, have assumed that the detectors in the network have identical sensitivity $[51,58,76,85]$.

The locations of future gravitational wave detectors have not yet been finalized. In this study we make use of the detector locations derived in [76] to optimize the location of future detectors. There, a three part figure of merit is used to determine the optimal location of detectors in a network, comprising equal parts: 1) how equally the network can determine both polarizations, 2) a simple measure of localization ability based on the area of the triangle formed by the detectors and 3) how accurately the chirp mass can be measured. The locations and orientations of all detectors are reported in the Appendix.

For the LIGO Voyager network, the location of the Hanford and Livingston detectors is fixed. Their orientations were chosen so that they were, as much as possible, sensitive to the same gravitational wave polarization, thus improving the chances of coincident detection [77]. The location of the LIGO India detector has not been announced at this time, so we use the optimal location from [76], which places it in a seismically quiet location. The triangular ET detector is added to this network to form the Voyager-ET network. In [76], it was shown that a location in Slovakia gave maximum flexibility when constructing a global network, so we choose this. Since ET is equally sensitive to both gravitational wave polarizations, the orientation of the detectors does not affect the results. It should be noted that the precise location in Europe of the triangular ET does not have a significant impact on results.

For the 3ET and CE networks, we are free to optimally site all three of the new detectors. In [76], with the additional requirements that the detectors lie on the land

\footnotetext{
${ }^{1}$ This does, however, lead to an increase in $40 \%$ in the length of tunnels required. In the case where the cost of constructing the tunnels is dominant, one could instead construct two $7.5 \mathrm{~km}$ interferometers within the same tunnel, making use of each tunnel twice as is done in the triangular ET design. In this scenario the tunnel length of the two L-shaped detectors is equal to the triangular configuration [108].
}

and avoid areas with a high degree of human activity, the authors arrived at two comparable networks for three triangular ET detectors. The best configurations had detectors in either Australia, Central Africa and the USA or in Australia, Europe and South America. Although the optimization was performed for triangular ET detectors, we use the first set of locations for both the 3ET and CE networks. We then optimize the orientation of the detectors based on part 1 of the figure of merit - sensitivity to both gravitational wave polarizations - as parts 2 and 3 will be largely insensitive to the orientation (this is described further in the Appendix). Finally, for the CE-ET network, we retain the two CE detectors in the USA and Australia and augment the network with a triangular ET detector in Europe.

This by no means covers the full set of proposed future detectors and networks, but is sufficient to allow us to explore the impact of a heterogeneous set of detector sensitivities and compare this to networks where all detectors have the same, or similar, sensitivity.

\section{Network sensitivity}

The response of a detector to the two polarizations of a gravitational wave is given by $F_{+}$and $F_{\times}$, which are functions of the sky location and polarization of the wave [109]. By writing the response time independently, we assume the strain is constant during the light round-trip time in the interferometer arms. For Voyager this is generally a good approximation. However for CE, and to a lesser extent ET, where the round-trips are respectively 20 and 5 times longer, the response pattern of a detector can differ in both amplitude and phase significantly from the static case for $\mathrm{GW}$ frequencies $\gtrsim 2 \mathrm{kHz}$ [110]. Nonetheless, for compact binary coalescences (CBCs) the vast majority of the signal-to-noise ratio (SNR) is accumulated at frequencies $\lesssim 2 \mathrm{kHz}$, and the static response is a good indication of sensitivity to these sources. We also do not account for the motion of the Earth. The loudest BNS signals could last hours to days in the detector bandwidth, introducing a time dependence in the detector response. This can be used to improve the localization, particularly when the network is operating with just one, or two, detectors and baseline triangulation is not possible [74,75]. However for the majority of signals this effect will be small.

For networks of equally sensitive detectors, the network response at a given sky point is given by $\left[\sum_{i}\left(F_{+}^{i}\right)^{2}+\left(F_{\times}^{i}\right)^{2}\right]^{1 / 2}[76,83]$. However, when dealing with heterogeneous networks, we must generalize the expression to take account of the detector sensitivity. To do so, we introduce a sensitivity measure $\sigma_{h, i}$ defined as $[111]^{2}$

\footnotetext{
${ }^{2}$ This quantity is typically often denoted $\sigma$. To avoid confusion with the signal bandwidth, $\sigma_{f}$, introduced in the next section, we have introduced the subscript $h$ here.
} 


$$
\sigma_{h, i}^{2}=4 \int_{0}^{\infty} \frac{\left|\tilde{h}_{o}(f)\right|^{2}}{S_{i}(f)} d f
$$

where $\tilde{h}_{o}(f)$ is the gravitational wave strain from a fiducial system placed overhead the detector at a fixed distance and face-on, and $S_{i}(f)$ is the power spectral density (PSD) of the detector noise. Then $\sigma_{h, i}$ gives the expected SNR for such a signal in detector $i$. For our study, we take $\tilde{h}_{o}(f)$ to be the signal from a face-on binary neutron star system at $1 \mathrm{Mpc}$ from the detector. Then, we weight the response of each detector by the sensitivity, defining [112]

$$
w_{+, \times}^{i}=\sigma_{h, i} F_{+, \times}^{i} .
$$

The relative sensitivity of the network at a given sky point is then defined as the network response,

$$
N_{R}=\left(\frac{\sum_{i}\left[\left(w_{+}^{i}\right)^{2}+\left(w_{\times}^{i}\right)^{2}\right]}{\sum_{j} \sigma_{w, j}^{2}}\right)^{1 / 2},
$$

where the indices $i, j$ run over the detectors. Using this definition, the maximum network response is unity, and this will only be achieved when all detectors are aligned to be maximally sensitive to the same sky position. This extends the definition of [83] to a heterogeneous network and is closely related to the network sensitivity to generic transients introduced in [82].

We are also interested in the relative sensitivity to the two gravitational wave polarizations. To define this unambiguously, we must identify a preferred choice of the + and $\times$ polarizations or, equivalently, a choice of a polarization angle. We define the dominant polarization frame $[112,113]$, which gives the maximum sensitivity to the + polarization. To do so, we introduce

$$
w_{+, \times}^{\text {net }}=\left(w_{+, \times}^{1}, \ldots, w_{+, \times}^{N}\right) .
$$

The dominant polarization frame, for a given sky location, is the unique frame such that: (1) $w_{\times}^{\text {net }} \cdot w_{+}^{\text {net }}=0$; (2) the network is maximally sensitive to the + polarization, thus ensuring $\left|w_{+}^{\text {net }}\right| \geq\left|w_{\times}^{\text {net }}\right|$. The ratio of $\left|w_{\times}^{\text {net }}\right|$ to $\left|w_{+}^{\text {net }}\right|$ is called the network alignment factor [113] and will vary from one-equal sensitivity to both polarizations-to zerosensitivity to a single polarization.

In Fig. 2, we plot the network response and alignment factor as a function of sky location for the five networks under consideration. The Voyager network has the best sensitivity above and below the location of the two US LIGO detectors, as expected. It has limited sensitivity to the second polarization over large parts of the sky, including the locations with best network sensitivity. In the VoyagerET network, the ET detector dominates the sensitivity so, as expected, we see the best sensitivity above and below the ET detector. The triangular ET is equally sensitive to both polarizations, and so the Voyager-ET network has good sensitivity to the second polarization over the majority of the sky. Even in regions where ET has poor sensitivity, the second polarization is reasonably well measured by a combination of ET and the three LIGO Voyager detectors.

The 3ET and 3CE networks are comprised of detectors in identical locations, so the relative sensitivity over the sky will be identical for these networks. These networks have good coverage over much of the sky, but the peak sensitivity is noticeably lower than the other networksit is only $75 \%$ of the maximum possible if all detectors were aligned, in comparison to over $90 \%$ for the other networks. This is to be expected, as the location of the detectors has been chosen to maximize sky coverage; three colocated detectors would provide the greatest peak sensitivity but much worse sky coverage. The homogeneous 3ET and 3CE networks have markedly better sensitivity to the second polarization than the Voyager network. This arises because the detector orientations were optimized to give good sensitivity to the second polarization, whereas the LIGO Hanford and Livingston detectors were deliberately aligned to be sensitive to the same polarization. Finally, the heterogeneous CE-ET network shows best sensitivity over the North Atlantic and Australia, which is expected given the detectors are located in the US, Europe and Australia. It has relatively poor sensitivity to the second polarization over the sky. However, in contrast to the Voyager network, CE-ET has good sensitivity to both polarizations in areas of good overall sensitivity.

The sky coverage [83] of a network is defined as the fraction of the sky for which the response is greater than $1 / \sqrt{2}$ of the maximum. The sky coverage of the homogeneous ET and CE networks is $79 \%$. Even though the LIGO Voyager network also has three equal sensitivity detectors, the similar orientations of the LIGO Hanford and Livingston detectors lead to a sky coverage of $42 \%$. For the heterogeneous CE-ET and Voyager-ET networks, the sky coverage is $44 \%$ and $37 \%$ respectively. This confirms what the plots suggest and indicates that the $3 \mathrm{ET}$ and $3 \mathrm{CE}$ networks have the most uniform response across the sky.

\section{SOURCE LOCALIZATION}

To investigate the ability of different networks to localize sources, we use the formalism introduced in Refs. [51,85] and references therein. In those papers, it was shown that localization is primarily determined by the timing accuracy, $\sigma_{t}$ in each detector which, in turn, is inversely proportional to the signal strength and frequency bandwidth $\sigma_{f}$ of the signal in the detector. Specifically, given a signal $h(t)$, the effective bandwidth is defined as

$\sigma_{f}^{2}=\left(\frac{4}{\rho^{2}} \int_{0}^{\infty} d f \frac{f^{2}|h(f)|^{2}}{S(f)}\right)-\left(\frac{4}{\rho^{2}} \int_{0}^{\infty} d f \frac{f|h(f)|^{2}}{S(f)}\right)^{2}$,

where the SNR, $\rho$, in the absence of noise, is given by 

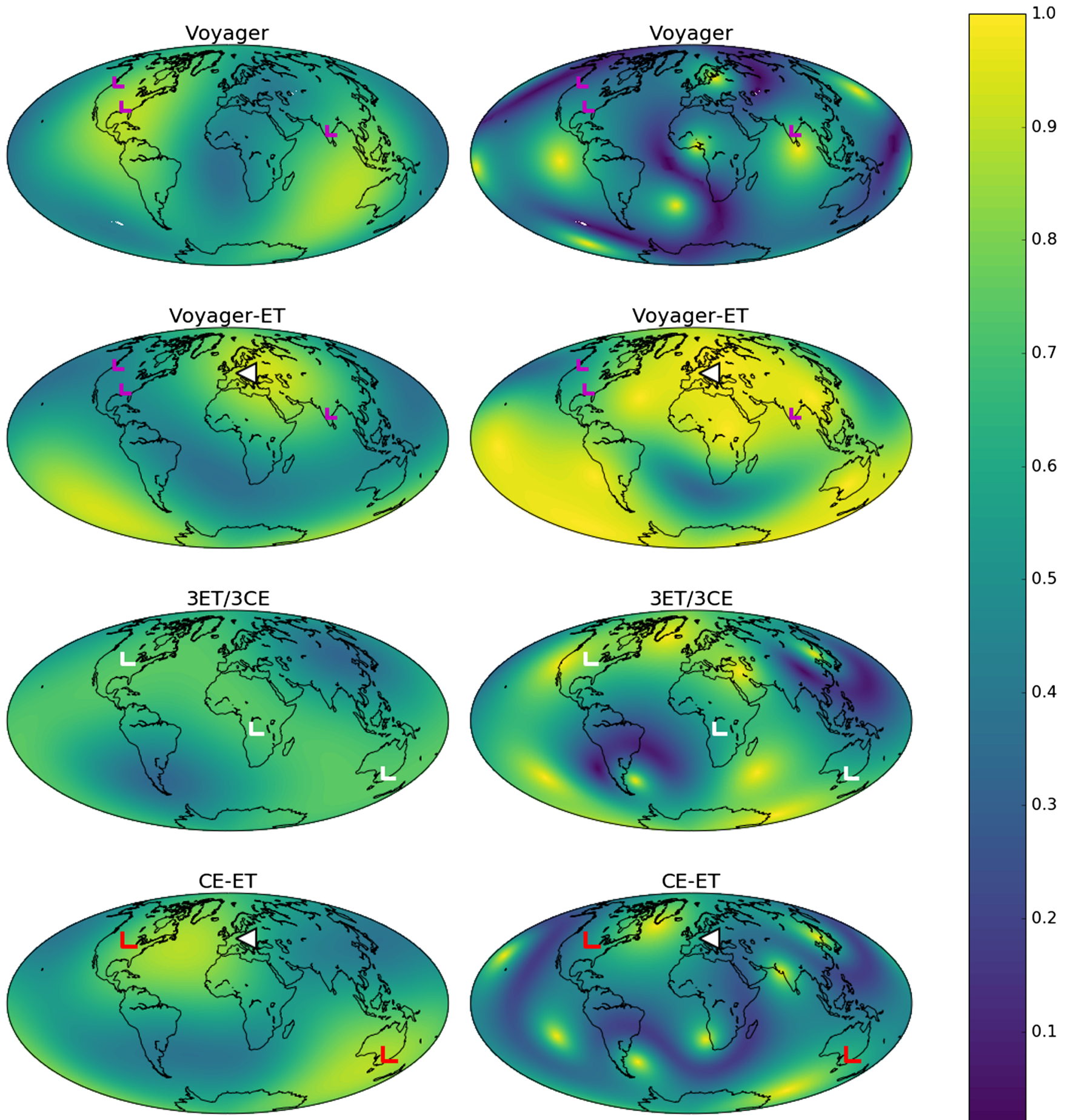

FIG. 2. Relative sensitivity of the different networks over the sky: Voyager, Voyager-ET, 3ET/3CE and CE-ET. Left: network response as a function of sky position and right: alignment factor as a function of sky position. Also shown are the locations of detectors in each network. Magenta markers are for Voyager detectors, white for ET, and red for CE. Since both $\mathrm{N}_{R}$ and the alignment factor are invariant under an overall scaling in network sensitivity with overall sensitivity of the network, 3ET and 3CE will have identical patterns.

$$
\rho^{2}=4 \int_{0}^{\infty} \frac{|h(f)|^{2}}{S(f)} d f .
$$

$$
\sigma_{t}=\frac{1}{2 \pi \rho \sigma_{f}} .
$$

The timing accuracy for a signal with SNR $\rho$ is then given by
Thus, $\sigma_{t}$ scales inversely with the SNR of the GW, $\rho$, and the effective bandwidth, $\sigma_{f}$, of the signal in the detector. 
Using these expressions, it is possible to calculate the reduction in network SNR due to errors in sky location and derive, at leading order, a relatively simple expression for the localization area. The probability distribution for the location of the source (from a sky location $\mathbf{R}$ ) is given by

$$
p(\mathbf{r} \mid \mathbf{R}) \propto p(\mathbf{r}) \exp \left[-\frac{1}{2}(\mathbf{r}-\mathbf{R})^{T} \mathbf{M}(\mathbf{r}-\mathbf{R})\right],
$$

where $\mathbf{r}$ is the reconstructed position of the source, $p(\mathbf{r})$ is the prior distribution (taken as uniform on the sphere), and the matrix $\mathbf{M}$ describes the localization accuracy and is given by

$$
\mathbf{M}=\frac{1}{\sum_{k} \sigma_{t_{k}}^{-2}} \sum_{i, j} \frac{\left(\mathbf{D}_{i}-\mathbf{D}_{j}\right)\left(\mathbf{D}_{i}-\mathbf{D}_{j}\right)^{T}}{2 \sigma_{t_{i}}^{2} \sigma_{t_{j}}^{2}}
$$

and $\mathbf{D}_{i}$ gives the location of the $i$ th detector. Thus, the localization is improved by having greater separation between the detectors and good timing accuracy, i.e., high SNR and large bandwidth of the signal in the detectors.

Localization can be improved by accounting for the relative amplitude and phase of the signal observed in each detector. These are necessarily constrained in a network of three or more detectors by the fact that a gravitational wave has only two polarizations. When taken into account, this leads to a more rapid falloff in the network SNR away from the correct sky location which, in turn, leads to an improvement in localization. This has been discussed in detail in [98], and a similar analysis was presented in [84]. The resulting probability distribution for the localization has the same form as Eq. (8) with a modified expression for the matrix $\mathbf{M}$, which nonetheless remains quadratic in the detector separations $\mathbf{D}_{i}-\mathbf{D}_{j}$.

Based on timing information alone, a source observed in three detectors can be localized to two regions in the sky. The two locations lie above and below the plane formed by the three detectors. When we require the signal to be consistent with two gravitational wave polarizations, this places restrictions on the relative amplitudes and phase differences between the detectors. In many cases, this information can be used to exclude the mirror location and restrict the source to a single sky position. Of course, with four or more sites, timing information alone can be used to localize a source to a single sky location.

In the following studies, we generate a population of events and determine which events would be detected by a given network and how accurately they would be localized. In all instances, we use the above formalism and ignore the effects of noise which would change the recovered SNR and offset the optimal sky location from the expected values. We require that signals would be confidently detected by the detector network. Specifically, we require a network SNR of at least 12 as well as an SNR above 5 in at least two detectors in the network. ${ }^{3}$ Furthermore, since the localization methods described above are accurate only to leading order, our localization results are based only upon detectors for which the signal has an SNR greater than 4. As discussed in [51], at lower SNRs the approximations used here break down. Due to these approximations, and our neglect of the changing detector response within a localization region, one should not expect exact agreement between our results and those obtained using existing codes (such as [86]). Detailed comparisons between our method and existing codes are planned in the future, see [98] for details.

The thresholds used mimic those used in the analysis of GW data [115] to obtain events with a false alarm rate of less than 1 per century $[85,86]$ and are the same as used in previous studies $[51,85]$. In addition, they seem appropriate based on the initial gravitational wave observations, where GW150914 and GW151226 both satisfied these requirements while LVT151012 had a network SNR of 10 and was not unambiguously identified as a signal [9]. As GW observations become more common, and searches are further improved [116], it is possible that the detection thresholds will be reduced. While this will change the details of the results presented below, the relative performance of the networks will remain similar.

\section{RESULTS}

\section{A. Face on binary neutron star mergers}

We first investigate the ability of the networks to localize a given source at a fixed distance, as a function of the sky location of a source. We simulate 1.4-1.4 $M_{\odot}$ BNS systems that are oriented face on (i.e., with inclination, $l=0$ ) at a fixed distance at each point along a two dimensional 16 by 16 grid of sky coordinates. We repeat the study for sources at redshifts of $z=0.2\left(D_{L}=1 \mathrm{Gpc}\right)$ and $z=0.5$ $\left(D_{L}=3 \mathrm{Gpc}\right)$. At each sky location, we calculate the expected SNR in each of the detectors in the network. For any signal that meets the detection and localization criteria given above, we calculate the $90 \%$ localization region. Since the BNS systems are face on, the GWs are circularly polarized, i.e., both polarizations have the same amplitude. Thus it is the overall sensitivity, and not the relative sensitivity to the two polarizations that will affect the localization ellipses [58]. This small set of sources with fixed distance and inclination allows for simple comparison with previous studies, e.g., [6,58]. Furthermore, for sources which are inclined at an angle $l \lesssim 45 \mathrm{deg}$, the relative amplitude of the two GW polarizations renders them indistinguishable from face-on signals $[98,117]$ and, indeed, the GW signals seen to date, including GW170817, have all been consistent with face-on binaries [49].

\footnotetext{
${ }^{3}$ For a discussion of the effects of changing these thresholds and, in particular, removing the single detector thresholds see [114].
} 
Figure 3 shows the localization regions for these BNS sources in the five networks under consideration. In the figures, a red cross indicates that the detection criteria (network SNR $>12$ and two detectors with SNR $>5$ ) were not met for a BNS at this sky position and redshift; a blue plus indicates that the source would be detected but fails our localization criterion (SNR $>4$ in three or more sites). For signals which would be confidently detected, and observed in at least three sites, the green ellipses show the $90 \%$ confidence region for the localization.

For BNS mergers at $z=0.2$, the LIGO Voyager network would observe the signal over the majority of the sky. There are, however, four patches where the signal would not be found, which correspond to areas of poor sensitivity for the two US LIGO detectors. Furthermore, there are regions where the signals would be detected but not localized, based on our conditions, and these correspond to locations where LIGO India has poor sensitivity. For those signals which are localized, the areas are typically large, as these events will be close to the detection threshold in the network. We can clearly identify a band for which the localizations are extended in one dimension. These points are close to the plane defined by the three detector locations. A large change in sky location, in a direction perpendicular to the plane of the detectors, leads to a relatively small change in the relative arrival times and consequently poor localization. These results are consistent with those obtained for the advanced LIGO network (incorporating LIGO India) given in [58].
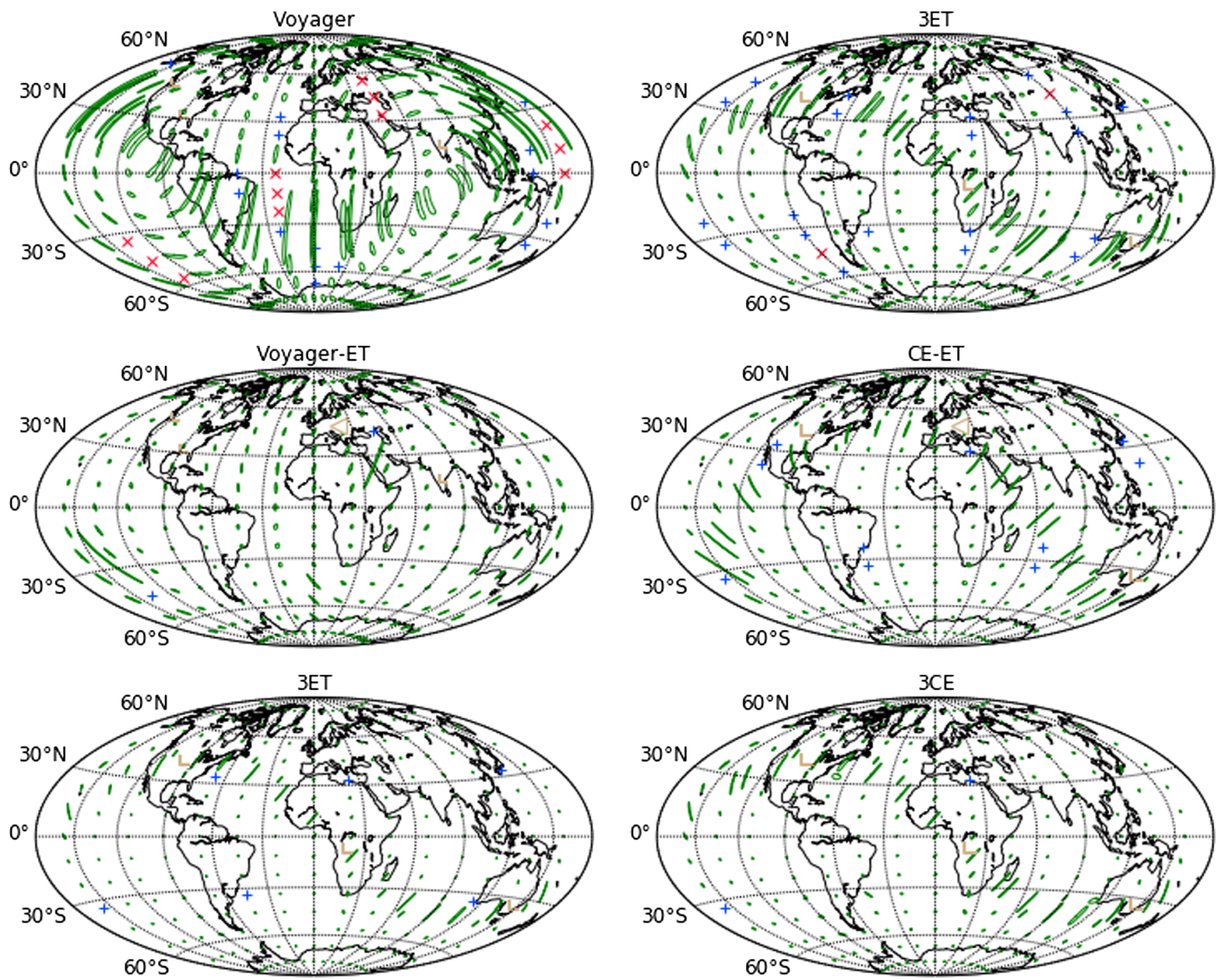

FIG. 3. The localization ellipses at different sky locations for face-on 1.4-1.4 BNS binaries at a redshift of: left- $z=0.2$ (luminosity distance of $1 \mathrm{Gpc}$ ) and right- $z=0.5$ (luminosity distance of $3 \mathrm{Gpc}$ ). The red crosses indicate that the BNS at this sky position would not be detected-either due to a network SNR less than 12, or not having SNR $>5$ in at least two detectors. The blue + symbols indicated sources that would be detected, but not well localized due to being identified in only two detectors. The ellipses give the $90 \%$ localization regions for a source from a given sky location. Detector locations are shown with golden markers. 
The Voyager-ET network is able to detect sources at $z=0.2$ over the essentially the whole sky. For localization, we require the signal to be observed at three sites; although all three of the detectors in the triangular ET will observe the signal, they provide rather poor localization by themselves. Thus, the network is limited by the requirement that two LIGO Voyager detectors observe the signal. The sky locations where sources are not localized correspond to the locations for which the US LIGO detectors have poor sensitivity, and these sources are only detected in ET and LIGO India. The 3ET network also gives excellent coverage over essentially the whole sky. There are still a handful of points for which localization is not possible. Again, these correspond to points where one of the detectors has close to zero sensitivity. As before, we see the characteristic extended ellipses at locations which lie close to the plane defined by the three ET detectors.

For signals at $z=0.5$ we consider the three networks comprised of ET and CE detectors. In all cases, the sources are observed over essentially the whole sky. For the 3ET network, there are significant regions where the source is not well localized as it is seen in only two detectors, but the size of these regions shrinks for the CE-ET and 3CE networks due to the increased sensitivity of the CE detector. Finally, as expected, the signals are relatively poorly localized in directions close to the plane defined by the three detectors.

For a two-site observation, the localization is typically restricted to a fraction of a ring in the sky with an area of hundreds of square degrees [2,9], and we consider these sources to not be localized. The degeneracy along the ring is broken by relative amplitude and phase measurements in the different detectors. For events observed with the triangular ET detector and a single L-shaped detector, the localization may be greatly improved-the triangular detector recovers the amplitude and phase of both GW polarizations so a single, additional observation will provide enough information to break the sky location degeneracy. Furthermore, when there are additional detectors in the network that did not observe the event, this information can be used to further improve the localization. We do not consider these effects here, but note that it would be interesting to examine in detail localization with a network comprised of one triangular and one L-shaped detector.

In these plots we are ignoring the fact that sources detected at three sites may be localized to two distinct patches in the sky, one above and one below the plane formed by the three sites. In many cases, the degeneracy can be broken based on consistency of the observed amplitude and phase of the signal in each of the detectors. For the systems at $z=0.2$, both the 3ET and Voyager-ET networks will provide localization to a single region for essentially all sky locations. Voyager localizes to one patch on the sky $70 \%$ of the time, and so about a third of the localization ellipses shown below will be augmented by a similar sized region in the mirror location. At $z=0.5$, the $\mathrm{CE}$ and ET networks all localize to a single patch for at least $95 \%$ of sky locations.

\section{B. A population of coalescing BNSs}

Now, let us consider network localization for a population of BNS coalescences. We follow Singer et al. [86] in choosing the BNS component masses uniformly in the astrophysically motivated range $1.2-1.6 M_{\odot}$. This encompasses the masses of all observed neutron stars in binaries and the 1-sigma interval of the initial mass function for a variety of formation mechanisms [118,119]. The orientation of the sources is uniformly distributed: uniform in polarization, cosine of source inclination and the phase of the $\mathrm{GW}$ at merger. We distribute the sources isotropically and assume their redshift distribution to follow the star formation rate density (SFRD) shifted to account for the delay between star birth and BNS merger [120], assuming standard cosmology [121],

$$
\frac{d R_{\mathrm{BNS}}}{d z}=\frac{d V}{d z} \frac{\Psi_{\mathrm{BNS}}(z)}{1+z},
$$

where $d V / d z$ is the differential comoving volume. The $(1+z)^{-1}$ factor accounts for the fact that a time interval $\Delta t_{z}$ in the rest-frame at $\mathrm{z}$ will be redshifted in the detector frame $(1+z) \Delta t$. The rest-frame merger rate density is given by

$$
\Psi_{\mathrm{BNS}}(z)=\int_{t_{*}}^{t(z)} \psi\left(z\left(t_{f}\right)\right) P\left(t-t_{f}\right) d t_{f},
$$

where $\psi\left(z\left(t_{f}\right)\right)$ is proportional to the observed cosmic SFRD [Eq. (15) from [122]], evaluated at the redshift the binary formed, and $P\left(t-t_{f}\right)$ encodes the delay time distribution. We assume the earliest epoch of star formation $t_{*}$ to be at $z_{f}=20$. The integral is normalized to give a local merger rate density of $\Psi_{\mathrm{BNS}}(0)=1500 \mathrm{Gpc}^{-3} \mathrm{y}^{-1}[3]$. We assume a distribution of possible delay times between star formation and BNS merger $P\left(t_{D}\right) \propto 1 / t_{D}$, and a minimum delay of $t_{D}^{\mathrm{min}}=0.2 \mathrm{Myr}$ corresponding to the smallest time a star can supernova and form a BNS [123]. The choice of $t_{D}^{\min }$ has little effect on the overall distribution. The $t_{D}^{-1}$ distribution of delay times is motivated both by population synthesis studies [123] and observations of merger times for six neutron star binaries [124] (see [125] and references therein).

Figure 4 shows the detection efficiency-the fraction of events that would be observed-for each network as a function of redshift or distance. For those BNS mergers which are detected by a given network, we calculate the 90\% confidence sky localization using the prescription given in Sec. III. We also show the fraction of events that 
would be localized within 1,10 and $100 \mathrm{deg}^{2}$ for each network as a function of redshift.

On the left-hand side of Fig. 4, we consider the Voyager, Voyager-ET and 3ET networks. The Voyager-ET and 3ET networks have rather comparable sensitivities, both networks identify over $90 \%$ of sources within a redshift of $z=0.2$ and the majority of signals within a redshift of $z=0.4$. The LIGO Voyager network has good all sky sensitivity within a redshift of $z=0.1$, after which it drops rapidly with essentially no sensitivity beyond $z=0.4$. Since we require a source to be observed in three sites for good localization, it is unsurprising that the Voyager and 3ET networks are capable of localizing the majority of observed sources-in particular, essentially all sources are localized within $100 \mathrm{deg}^{2}$ and the majority within $10 \mathrm{deg}^{2}$. For the heterogeneous Voyager-ET network, the fraction of sources localized is much lower than the fraction detected. For example, at $z=0.4$ over half of all sources are detected but only $10 \%$ are localized within $10 \mathrm{deg}^{2}$. These are the events which are too distant to be observed by the LIGO Voyager detectors so, while they are observed by ET they cannot be localized. For all three networks, only a fraction of events will be localized to within $1 \mathrm{deg}^{2}$, and those will be primarily nearby, loud events. For a 3ET network, half of the events at a redshift of $z=0.15$ with be localized to within $1 \mathrm{deg}^{2}$.

The right panel of Fig. 4 shows the same results for the $\mathrm{CE}$ and ET based networks. The results are comparable to those described above: detection efficiency is limited by the second most sensitive detector in the network, while localization requires a third detector to observe the signal. In particular, we note that while the CE-ET and $3 \mathrm{CE}$ networks have similar overall detection efficiencies, the $3 \mathrm{CE}$ network provides much better localizations. For example, 3CE localizes $50 \%$ of sources at $z=2$ to $100 \mathrm{deg}^{2}$ while the CE-ET network is unable to give good localizations for signals at this redshift. We note that those signals which are localized in the heterogeneous CE-ET network are typically localized within $10 \mathrm{deg}^{2}$ as they will be recovered with high SNR in the Cosmic Explorer detectors. Finally, it is again only the loudest, nearby signals which are localized within $1 \mathrm{deg}^{2}$. The CE-ET and 3CE networks localize half of signals within $1 \mathrm{deg}^{2}$ to a redshift of $z \sim 0.25$.

Figure 5 shows the expected number of observed events as a function of redshift for the five networks, and the overall results are summarized in Table I. In order to obtain these results, we have taken a population of sources assumed to follow the star formation rate. While the star formation rate is reasonably well constrained up to its peak at a redshift of one to two, observational evidence at higher redshifts, and for the distribution of delay times between binary formation and merger, is relatively scarce. Furthermore, we use an intrinsic merger rate of $1500 \mathrm{Gpc}^{-3} \mathrm{y}^{-1}$, which lies within the current range of predicted rates [3], and we use this fiducial rate when
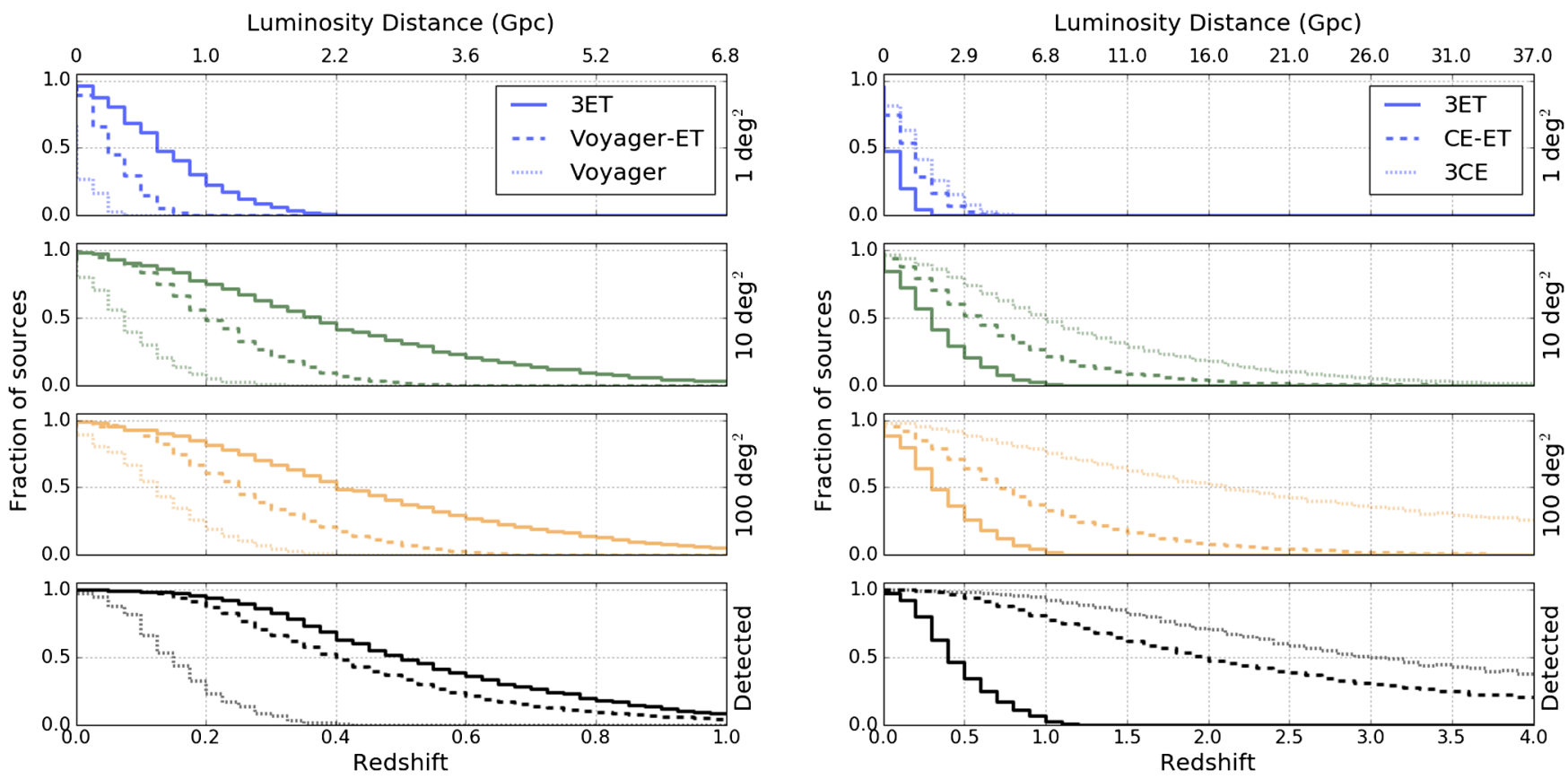

FIG. 4. The detection and localization efficiency as a function of redshift and luminosity distance of the Voyager, Voyager-ET and 3ET networks (left column) and the 3ET, CE-ET and 3CE networks (right column). For visual comparison, 3ET is plotted as a solid line in both. Left column: Voyager-ET and Voyager are the dashed and dotted lines respectively. Right column: CE-ET and 3CE are the dashed and dotted lines respectively. From bottom to top, the rows show the fraction of events at a given redshift/distance that will be detected and localized within 100, 10 and $1 \mathrm{deg}^{2}$. 

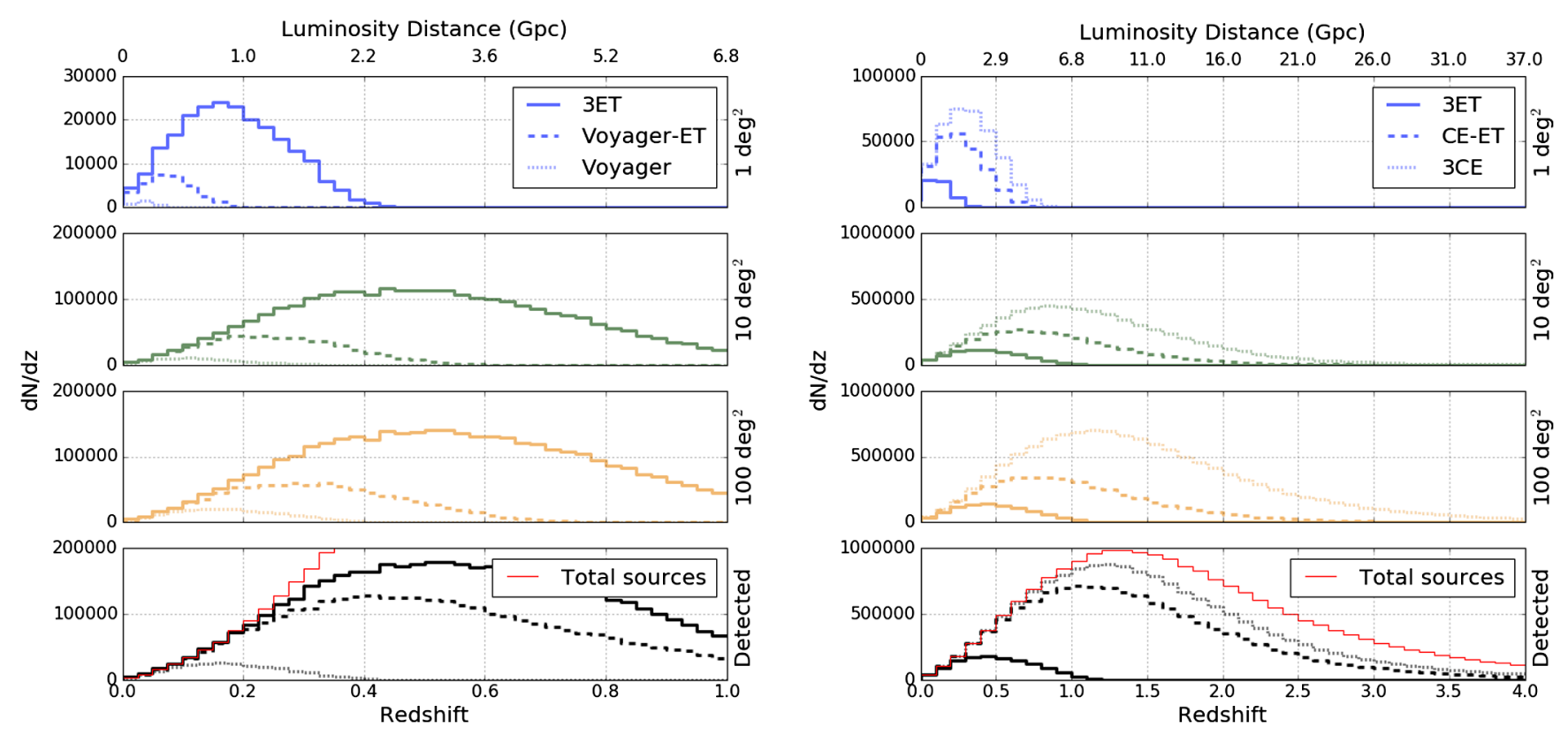

FIG. 5. The number of BNS observations and localizations per year with future networks, as a function of redshift and luminosity distance. The $\mathrm{y}$-axis is scaled so that the area under the curves gives the number of events per year. This assumes an isotropic redshift distribution that follows the star formation rate [122] shifted to account for the delay between star birth and BNS merger [120] and a distribution of possible delay times $P\left(t_{D}\right) \propto 1 / t_{D}$, with a minimum delay of $t_{D}^{\min }=0.2$ Myr [123]. The local merger rate density is taken as $\Psi_{\mathrm{BNS}}(0)=1500 \mathrm{Gpc}^{-3} \mathrm{y}^{-1}$ [3]. Note that the y-axis on $1 \mathrm{deg}^{2}$ plot is different from the others. For visual comparison 3ET is plotted as a solid line in both column. Left column: Voyager-ET and Voyager are the dashed and dotted lines respectively. Right column: CE-ET and $3 \mathrm{CE}$ are the dashed and dotted lines respectively. From bottom to top, the rows show the number density of events at a given redshift/ distance that will be detected and localized within 100, 10 and $1 \mathrm{deg}^{2}$.

comparing the network sensitivities. There is at least an order of magnitude uncertainty on the rate of BNS mergers. Overall changes to the merger rate will simply scale the number of observations for all networks equally but changes in the redshift evolution of the rate will affect relative performances.

As expected, the number of events detected by the 3ET and Voyager-ET networks are comparable. The 3ET network is sensitive to $50 \%$ more BNS mergers than Voyager-ET, while the Voyager network observes less than a tenth as many mergers. However, due to the differences in localization, the Voyager-ET localizes only a quarter as many events as the 3ET network and, for events localized within $10 \mathrm{deg}^{2}$ the peak of the redshift distribution is at 0.2 rather than 0.4 (and within $1 \mathrm{deg}^{2}$ the peak is at 0.06 rather than 0.2). We see similar results for the CE-ET and $3 \mathrm{CE}$ networks: they are both able to detect a comparable number of events, but significantly fewer are localized by the heterogeneous network.

For the results presented above, we have assumed a $100 \%$ duty cycle for all detectors in the network. Here we consider how the results would change for a more realistic

TABLE I. Performance metrics for Voyager, Voyager-ET, 3ET, CE-ET and 3CE networks for a population of BNS coalescences distributed uniformly in comoving volume with an intrinsic merger rate of $1500 \mathrm{Gpc}^{-3} \mathrm{y}^{-1}$. From top to bottom: The number of sources per year that are detected and localized by each network; the number of sources localized per year within 1 , 10 and 100 deg $^{2}$ respectively; the median localization area of all detected sources and the fraction of localized sources whose position is restricted to a single patch in the sky. Note, the median source is not localized by the two heterogeneous networks CE-ET and Voyager-ET.

\begin{tabular}{lcccrr}
\hline \hline Network & Voyager & Voy-ET & 3ET & CE-ET & 3CE \\
\hline Detected & 6000 & 83000 & 120000 & 1300000 & 1600000 \\
Localized & 5500 & 23000 & 100000 & 470000 & 1400000 \\
Within 1 deg & 100 & 830 & 5600 & 24000 & 37000 \\
Within $10 \mathrm{deg}^{2}$ & 1900 & 14000 & 73000 & 300000 & 650000 \\
Within $100 \mathrm{deg}^{2}$ & 4800 & 23000 & 95000 & 450000 & 1300000 \\
Median area $\left(\mathrm{deg}^{2}\right)$ & 22 & $\ldots$ & 7 & $\ldots$ & 13 \\
Single patch & $45 \%$ & $99 \%$ & $84 \%$ & $97 \%$ & $90 \%$ \\
\hline \hline
\end{tabular}


observing scenario. Experience from previous observing runs tell us that at design sensitivity duty cycle for an individual detector is about $80 \%$, although it is possible that this may be different for third-generation detectors. Thus, we assume an $80 \%$ duty cycle for all detectors in the network, with uncorrelated operating times between the detectors, with the exception of the triangular ET where we assume perfect correlation of up-time between the interferometers. For the three detector networks, this means that roughly half the time all three detectors will be operational. For an additional $40 \%$ of the time, two detectors will be operating and they will, on average, be sensitive to half as many signals as the three detector network and, based on our criteria for localization, not localize any of them. With the four site Voyager-ET network, we will have all four detectors operating $40 \%$ of the time. There will then be $10 \%$ of the time when the three Voyager detectors are operating, and an additional 30\% when ET plus two of the Voyager detectors are running. Localization with ET and two Voyagers will vary depending on which Voyager detector is off-line. For instance, a network of the triangular ET augmented by the two American Voyagers at Hanford and Livingston would localize $70 \%$ as many within $100 \mathrm{deg}^{2}$ as Voyager-ET but only provide $30 \%$ as many subsquare-degree localizations. This is due to the fact that the alignment of Hanford and Livingston maximises the chances of the network meeting the localization criteria, but coupled with the smaller baseline between these detectors, the alignment also limits the precision of the localization. On the other hand, when one of the American Voyager detectors is off-line, only about half of the number will be localized within 10 and $100 \mathrm{deg}^{2}$ as Voyager-ET, with 60\% $(40 \%)$ as many subsquare-degree localizations when the Livingston (Hanford) detector is off-line.

\section{Implication for EM follow-up}

The observation of broadband electromagnetic emission associated with GW170817 provided conclusive evidence that BNS mergers are a progenitor of short gamma ray bursts (GRB), and also power kilonova emissions [23-46]. The primary motivation for accurate localization of future GW signals is to facilitate the observation of electromagnetic counterparts. The requirements on localization will depend upon the strength of the electromagnetic emission accompanying a BNS merger, as well as the ability of widefield telescopes to cover the error region. While short GRBs can be observed to cosmological distances, they are believed to be rather tightly beamed, so that only a small fraction of BNS mergers would be accompanied by a gamma ray burst (GRB) counterpart [126]. However, observations of GW170817 imply that the gamma ray burst (GRB) emission is structured [25] with a broader, weak emission, possibly powered by a cocoon $[47,127,128]$. Since the gamma ray burst (GRB) emission is likely to be essentially concurrent with the merger, it will be difficult to use GW observations to provide advanced warning to gamma ray burst (GRB) satellites. On the other hand, kilonova emission is expected to last for days or possibly weeks in the optical and near infrared bands $[129,130]$. Furthermore while gamma ray burst (GRB) are beamed, kilonovae are relatively isotropic, and thus are the more likely counterpart to a typical BNS merger [18]. We therefore focus the rest of the discussion on kilonovae.

The neutron-rich ejecta from BNS and NSBH mergers will undergo r-process nucleosynthesis, producing heavy elements which will subsequently decay; this decay process will power an electromagnetic transient known as a kilonova (see e.g., [131] for details). There are various models for the kilonova emission, which depend upon the mass of the ejecta as well as its opacity $[129,130]$. Broadly, the prediction is for an optical or near infrared emission, which will last for days or possibly weeks. The luminosity of the kilonova emission is uncertain, but we take a fiducial model with magnitude 22 emission from a source at $400 \mathrm{Mpc}$, equivalent to the kilonova emission from GW170817 [132]. To date, there has been one other putative nearinfrared kilonova observation from gamma ray burst (GRB) 130603B which was observed with a magnitude of 25.8 at redshift of $z \approx 0.35$ (equivalently 22.5 at $400 \mathrm{Mpc}$ ) $[133,134]$, which is broadly consistent with this picture.

Taking our fiducial kilonova model, the current generation of wide-field telescopes, such as DECam [29], PanSTARRS [39] and VISTA [40], which have limiting magnitudes around 22 would be able to observe kilonova emission to $z \approx 0.1$ or a luminosity distance of $400 \mathrm{Mpc}$. The results in Figs. 4 and 5 show that the Voyager network has good sensitivity within the range of the current generation of telescopes, and would identify and localize the majority of BNS mergers at $z \lesssim 0.1$ to within $10 \mathrm{deg}^{2}$. All of the other networks are able to detect, and localize within $10 \mathrm{deg}^{2}$, essentially every event at $z \lesssim 0.1$, thereby enabling follow-up with one, or a handful, of pointings.

For LSST [135], with a limiting magnitude around $25-26$, kilonovae could be observed to $z \approx 0.4$ or $2 \mathrm{Gpc}$. At these distances, the sensitivity of the Voyager network is sufficient to identify only a minority, let alone provide accurate localizations. The Voyager-ET network would observe the majority of BNS within this range. However, the more distant signals would be observed only by the ET detector and consequently be poorly localized. Again using the fiducial kilonova model, around one third of BNS mergers producing a kilanova observable by LSST would be localized within $10 \mathrm{deg}^{2}$. A 3ET network would identify a similar number of mergers, and localize twice as many LSST-observable kilonovae as the Voyager-ET network. The networks with CE detectors are complete with LSST's kilonova horizon, and localize the vast majority of sources to within $10 \mathrm{deg}^{2}$.

Of course, the details of kilonova emission are still uncertain and there are models that predict significantly 
stronger or weaker emission. For example models, typically with a smaller ejecta mass, predict magnitude 22 emission at $200 \mathrm{Mpc}$ [131]. In this case the Voyager network would be sufficient to detect and localize the majority of signals within the range of the current generation of telescopes. The Voyager-ET network would localize the majority of signals within the LSST range for these signals. Thus, the case for localization capacity of future GW networks remains intimately tied to our knowledge of the range of EM emission from these mergers.

As some of the strongest emissions are predicted from NSBH mergers, it is interesting to briefly consider them. While we have not performed simulations with NSBH systems, it is straightforward to provide approximate sensitivities based on the BNS results given above. The sensitivity of gravitational wave detectors scales, at leading order, as $\mathcal{M}^{5 / 6}$, where $\mathcal{M}$ is the chirp mass. Consequently, for a signal at a fixed distance, orientation and sky location, the SNR with which NSBH will be observed can be approximated as

$$
\rho_{\mathrm{NSBH}} \approx 1.1\left(\frac{M_{\mathrm{BH}}}{M_{\mathrm{NS}}}\right)^{1 / 2}\left(1+\frac{M_{\mathrm{BH}}}{M_{\mathrm{NS}}}\right)^{-1 / 6} \rho_{\mathrm{BNS}},
$$

where $M_{\mathrm{BH}}$ is the black hole mass and $M_{\mathrm{NS}}$ is the neutron star mass. Thus, the observed SNR for a NSBH with $M_{\mathrm{BH}}=5 M_{\odot}$ is 1.6 times that of a BNS, and 2.1 times for $M_{\mathrm{BH}}=10 M_{\odot}$. Consequently, to a reasonable approximation, we can scale the distances in Fig. 4 by these factors to obtain NSBH sensitivities.

A network's localization accuracy to NSBHs at a given distance will be improved relative to BNSs, due to higher SNR but will be reduced due to lower frequency bandwidth [as defined in Eq. (5)]. The effective bandwidth of a NSBH binary with a $5 M_{\odot}\left(10 M_{\odot}\right)$ black hole is $10 \%(30 \%-40 \%)$ less than a BNS. Thus localization areas will be roughly a factor of $1.5^{2}$ smaller for NSBH than BNS at a given distance. So, we can approximate NSBH localizations by rescaling the distances in Fig. 4 by a factor of 1.5.

For the most optimistic NSBH kilonova emissions, which predict magnitude 20 emission at $200 \mathrm{Mpc}$ powered by fallback accretion [131], a network with three ET or CE detectors would identify and localize most sources within the LSST range; Voyager augmented by ET would identify but not localize the more distant sources and Voyager alone would have a range comparable to existing wide-field telescopes.

\section{DISCUSSION}

We have compared the sensitivity of proposed future gravitational wave networks to BNS signals and their ability to accurately localize these events. We find that a minimum of two detectors, which includes the triangular ET, at an improved sensitivity are sufficient to provide a substantial increase in the number of observed sources. For example, the addition of ET to a network of LIGO-Voyager sensitivity detectors could increase the rate of observations by an order of magnitude. However, in order to obtain good source localization, we require a minimum of three sites to observe the event. Consequently, in networks with one or two detectors that are significantly more sensitive than the others, we find that the majority of detected sources are not well localized. In contrast, when the three most sensitive detectors in the network have comparably sensitivity, the majority of signals are well localized with a median localization area around $10 \mathrm{deg}^{2}$.

Previously it has been argued that building more detectors further apart improves localization (see for example $[82,85])$. However, we find that this is only true when the sensitivities of the detectors in the network are approximately homogeneous, as is often assumed for the advanced detector (second generation) networks $[51,58,85]$. In the case of heterogeneous sensitivities, such as the Voyager-ET network, the localization will often be limited since the event cannot be detected by the less sensitive detectors. In such a network, we expect that for a majority of events we will obtain limited directional information.

The interpretation of our results depends critically upon the science question of interest. In particular, the utility of accurate GW localization as a function of redshift will depend upon the strength and spectrum of the associated EM emission, and the sensitivity and field of view of the associated telescopes and satellites. For a standard kilonova model, the LIGO Voyager network provides adequate sensitivity to identify and localize potential kilonova signals for currently operating telescopes but the network must be augmented by at least one ET or CE detector to provide adequate sensitivity to localize all kilonovae that could be observed by LSST. For the models predicting the strongest kilonova emission, a three detector network of ET or CE detectors could increase, by a factor of a few, the number of events observed jointly with GW signals.

In this study, we have neglected a number of factors that affect the size of localization errors in real detector networks. Though the time of arrival and amplitude and phase of GWs carry most of the information relevant to localization, other information can reduce the size of the localization errors. These include, realistic prior distributions on other astrophysical parameters-particularly source inclination and distance, correlations with other parameters such as component masses [97], spin and precession effects. Furthermore, we continue to assume that a signal must be identified in two detectors to be detected, and three to be localized. Ideally, performing a fully coherent analysis of the data $[114,136]$, would improve the performance of heterogeneous networks when the SNR in the less sensitive detectors is low and would, in effect, remove our requirement of a signal being clearly identified in at least two detectors. While it is possible to localize sources with only two detectors, the first GW 
observations make it clear that the localization areas will typically be hundreds of square degrees, so our approximation that these sources are not localized is reasonable. However, incorporation of nondetection (or weak detection) in additional detectors, as was done for GW170817 [3], can lead to improved localizations.

We have neglected systematic uncertainties in the results presented here. Errors introduced by mismatches between template waveforms and signals [51] are expected to introduce a similar effect in all detectors, and therefore the effect on the time difference, and localization, is likely to be negligible. On the other hand, errors in the calibration of GW detectors [137] will be uncorrelated, and these errors can significantly impact localization. For instance, roughly one third of the localization error budget for GW150914 was due to strain calibration uncertainty [9,137]. At high SNR, calibration errors are expected to dominate the overall error budget for localization [51]. Thus, the ability to achieve the reported subsquare-degree localizations predicted here will depend critically on the calibration accuracy of the detectors, with likely requirements of uncertainties under $1 \%$ in amplitude and $1^{\circ}$ in phase. The impact of calibration on gravitational wave localization with future networks deserves further study.

Finally, in this paper we have restricted attention to localization of BNS signals. For many science questions, accurate localization itself is not critical but is required for accurate measurement of the distance to the source. The gravitational wave signal from the inspiral and merger of BNS, NSBH and BBH leads to the accurate measurement of only the luminosity distance $D_{L}$ and the redshifted masses $M(1+z)$. One goal of gravitational wave astronomy is to map the merger history of black holes and neutron stars through cosmic time. Accurate distance measurements are required not only to infer the redshift of the source, but also to obtain the mass of the source. For tests of cosmology, we require an independent measurement of the redshift. There are numerous methods proposed for this measurement, including identification of a host galaxy from EM counterpart; statistical association with a host galaxy [138]; assumption of a narrow mass range of neutron stars in binaries [125]; observation of postmerger features in the waveform [139]. In all cases, an accurate measurement of the distance (and consequently good source localization) is essential. A detailed investigation of these issues is beyond the scope of this paper. Nonetheless, it seems likely that a network of three or more detectors of comparable sensitivity will increase scientific returns from a future gravitational wave network.

\section{ACKNOWLEDGMENTS}

The authors would like to thank Rana Adhikari, Lisa Barsotti, Yanbei Chen, Matt Evans, Laura Nuttall, Bangalore Sathyaprakash, Patrick Sutton, and Salvatore Vitale for useful discussions. Also the authors are grateful to Tito Dal Canton for many helpful suggestions to improve the presentation of the paper. This work was supported by STFC Grant No. ST/N005430/1 and ERC grant Ref 647839.

\section{APPENDIX: OPTIMAL DETECTOR ORIENTATIONS}

Table II contains all the final locations and orientations of all detectors used in this study. In order to fix the orientations of the detectors for the ET and CE networks, we make use of the following FoM, taken from Ref. [76]:

$$
I=\left(\frac{1}{4 \pi} \oint\left|w_{+}^{\text {net }}-w_{\times}^{\text {net }}\right|^{2} d \Omega\right)^{-1 / 2} .
$$

Holding the USA detector fixed at $0^{\circ}$ we rotated the Central Africa and Australia detectors from $0^{\circ}-90^{\circ}$ (due to rotational symmetry of the polarizations all other rotations map to this basis). We optimize the ability of the ET and CE networks to observe both polarizations by choosing the orientation angles to maximize (A1).

TABLE II. The locations and orientations of the detectors used in this study. All numbers are given in degrees and the orientation angle is defined clockwise relative to a hypothetical L-shaped interferometer with arms due North and East.

\begin{tabular}{lccc}
\hline \hline Detector & Longitude & Latitude & Orientation \\
\hline LIGO Livingston Voyager & -90.77 & 30.56 & 162.3 \\
LIGO Hanford Voyager & -119.41 & 46.46 & 234.0 \\
LIGO India Voyager & 76.4 & 14.2 & 346.8 \\
European $\Delta$ ET & 18.7 & 48.5 & $\ldots$ \\
Central Africa CE/ET & 17.2 & -9.9 & 82.4 \\
Australia CE/ET & 146.9 & -35.8 & 84.4 \\
USA CE/ET & -98.4 & 38.9 & 0 \\
\hline \hline
\end{tabular}

[1] A. Einstein and K. Sitzungsber, , Über Gravitationswellen, Preuss. Akad. Wiss. 1, 688 (1916).

[2] B. P. Abbott et al., Observation of Gravitational Waves from a Binary Black Hole Merger, Phys. Rev. Lett. 116, 061102 (2016).
[3] B. P. Abbott et al., Gw170817: Observation of Gravitational Waves from a Binary Neutron Star Inspiral, Phys. Rev. Lett. 119, 161101 (2017).

[4] J. Aasi et al. (LIGO Scientific Collaboration), Advanced LIGO, Classical Quantum Gravity 32, 074001 (2015). 
[5] F Acernese et al., Advanced virgo: A second-generation interferometric gravitational wave detector, Classical Quantum Gravity 32, 024001 (2015).

[6] B. P. Abbott et al., Prospects for observing and localizing gravitational-wave transients with Advanced LIGO and Advanced Virgo, Living Rev. Relativity 19, 1 (2016).

[7] B.S. Sathyaprakash et al., Scientific benefits of moving one of LIGO Hanford detectors to India, Technical Report, http://www.ligo.caltech.edu/NSF/related/10.2011/ ligo-india-110923.pdf.

[8] Y. Aso, Y. Michimura, K. Somiya, M. Ando, O. Miyakawa, T. Sekiguchi, D. Tatsumi, and H. Yamamoto, Interferometer design of the KAGRA gravitational wave detector, Phys. Rev. D 88, 043007 (2013).

[9] B. P. Abbott et al., Binary Black Hole Mergers in the First Advanced LIGO Observing Run, Phys. Rev. X 6, 041015 (2016).

[10] B. P. Abbott et al., Upper limits on the rates of binary neutron star and neutron star-black hole mergers from Advanced Ligo's first observing run, Astrophys. J. 832, L21 (2016).

[11] B. P. Abbott et al., All-sky search for short gravitationalwave bursts in the first Advanced LIGO run, Phys. Rev. D 95, 042003 (2017).

[12] B. P. Abbott et al., Search for gravitational waves associated with gamma-ray bursts during the first Advanced LIGO observing run and implications for the origin of GRB 150906B, Astrophys. J. 841, 89 (2016).

[13] B. P. Abbott et al., Localization and broadband follow-up of the gravitational-wave transient GW150914, Astrophys. J. 826, L13 (2016).

[14] E. Chassande-Mottin, M. Hendry, P. J. Sutton, and S. Márka, Multimessenger astronomy with the Einstein Telescope, Gen. Relativ. Gravit. 43, 437 (2011).

[15] P. S. Cowperthwaite and E. Berger, A comprehensive study of detectability and contamination in deep rapid optical searches for gravitational wave counterparts, Astrophys. J. 814, 25 (2015).

[16] J. Kanner, T. L. Huard, S. Márka, D. C Murphy, J. Piscionere, M. Reed, and P. Shawhan, Looc up: Locating and observing optical counterparts to gravitational wave bursts, Classical Quantum Gravity 25, 184034 (2008).

[17] H. M. Lee et al., Gravitational wave astrophysics, data analysis and multimessenger astronomy, Sci China-Phys Mech Astron 58, 120403 (2015).

[18] B. D. Metzger and E. Berger, What is the most promising electromagnetic counterpart of a neutron star binary merger?, Astrophys. J. 746, 48 (2012).

[19] S. Nissanke, M. Kasliwal, and A. Georgieva, Identifying elusive electromagnetic counterparts to gravitational wave mergers: An end-to-end simulation, Astrophys. J. 767, 124 (2013).

[20] L. K. Nuttall and P. J. Sutton, Identifying the host galaxy of gravitational wave signals, Phys. Rev. D 82, 102002 (2010).

[21] C. W. Stubbs, Linking optical and infrared observations with gravitational wave sources through transient variability, Classical Quantum Gravity 25, 184033 (2008).
[22] J. Sylvestre, Prospects for the detection of electromagnetic counterparts to gravitational wave events, Astrophys. J. 591, 1152 (2003).

[23] B. P. Abbott et al., Multi-messenger observations of a binary neutron star merger, Astrophys. J. Lett. 848, L12 (2017).

[24] B. P. Abbott et al., Gravitational waves and gamma-rays from a binary neutron star merger: GW170817 and GRB 170817a, Astrophys. J. 848, L13 (2017).

[25] A. Goldstein et al., An ordinary short gamma-ray burst with extraordinary implications: Fermi -GBM detection of GRB 170817a, Astrophys. J. 848, L14 (2017).

[26] V. Savchenko et al., INTEGRAL Detection of the first prompt gamma-ray signal coincident with the gravitational wave event GW170817, Astrophys. J. 848, L15 (2017).

[27] D. A. Coulter et al., Swope supernova survey 2017a (SSS17a), the optical counterpart to a gravitational wave source, Science 358, 1556 (2017).

[28] M. Soares-Santos et al., The electromagnetic counterpart of the binary neutron star merger LIGO/Virgo GW170817. I. Dark energy camera discovery of the optical counterpart, Astrophys. J. 848, L16 (2017).

[29] P. S. Cowperthwaite et al., The electromagnetic counterpart of the binary neutron star merger LIGO/VIRGO GW170817. II. UV, optical, and near-IR light curves and comparison to kilonova models, Astrophys. J. 848, L17 (2017).

[30] M. Nicholl et al., The electromagnetic counterpart of the binary neutron star merger LIGO/VIRGO GW170817. III. Optical and UV spectra of a blue kilonova from fast polar ejecta, Astrophys. J. 848, L18 (2017).

[31] R. Chornock et al., The electromagnetic counterpart of the binary neutron star merger LIGO/VIRGO GW170817. IV. Detection of near-infrared signatures of r-process nucleosynthesis with gemini-south, Astrophys. J. 848, L19 (2017).

[32] R. Margutti et al., The electromagnetic counterpart of the binary neutron star merger LIGO/VIRGO GW170817. V. Rising $\mathrm{x}$-ray emission from an off-axis jet, Astrophys. J. 848, L20 (2017).

[33] K. D. Alexander et al., The electromagnetic counterpart of the binary neutron star merger LIGO/VIRGO GW170817. VI. Radio constraints on a relativistic jet and predictions for late-time emission from the kilonova ejecta, Astrophys. J. 848, L21 (2017).

[34] P. K. Blanchard et al., The electromagnetic counterpart of the binary neutron star merger LIGO/VIRGO GW170817. VII. Properties of the host galaxy and constraints on the merger timescale, Astrophys. J. 848, L22 (2017).

[35] W. Fong, E. Berger et al., The electromagnetic counterpart of the binary neutron star merger LIGO/VIRGO GW170817. VIII. A comparison to cosmological shortduration gamma-ray bursts, Astrophys. J. 848, L23 (2017).

[36] I. Arcavi et al., Optical emission from a kilonova following a gravitational-wave-detected neutron-star merger, Nature (London) 551, 64 (2017).

[37] M. C. Diaz et al., Observations of the first electromagnetic counterpart to a gravitational wave source by the TOROS collaboration, Astrophys. J. 848, L29 (2017). 
[38] V.M. Lipunov et al., MASTER optical detection of the first LIGO/Virgo neutron stars merging GW170817, Astrophys. J. 850, L1 (2017).

[39] S. J. Smartt et al., A kilonova as the electromagnetic counterpart to a gravitational-wave source, Nature (London) 551, 75 (2017).

[40] N. R. Tanvir et al., The emergence of a lanthanide-rich kilonova following the merger of two neutron stars, Astrophys. J. 848, L27 (2017).

[41] Y. Utsumi et al., J-GEM observations of an electromagnetic counterpart to the neutron star merger GW170817, Publ. Astron. Soc. Jpn. 69, 101 (2018).

[42] S. Valenti, D. J. Sand, S. Yang, E. Cappellaro, L. Tartaglia, A. Corsi, S. W. Jha, D. E. Reichart, J. Haislip, and V. Kouprianov, The discovery of the electromagnetic counterpart of GW170817: kilonova AT 2017gfo/DLT17ck, Astrophys. J. 848, L24 (2017).

[43] P. A. Evans et al., Swift and NuSTAR observations of GW170817: detection of a blue kilonova, Science 358, 1565 (2017).

[44] M. R. Drout et al., Light curves of the neutron star merger GW170817/SSS17a: Implications for r-process nucleosynthesis, Science 358, 1570 (2017).

[45] E. Troja et al., The X-ray counterpart to the gravitational wave event GW 170817, Nature (London) 551, 71 (2017).

[46] G. Hallinan et al., A Radio counterpart to a neutron star merger, Science 358, 1579 (2017).

[47] M. M. Kasliwal et al., Illuminating gravitational waves: A concordant picture of photons from a neutron star merger, Science 358, 1559 (2017).

[48] D. Kasen, B. Metzger, J. Barnes, E. Quataert, and E. Ramirez-Ruiz, Origin of the heavy elements in binary neutron-star mergers from a gravitational-wave event, Nature (London) 551, 80 (2017).

[49] LIGO Scientific Collaboration and Virgo Collaboration, 1M2H Collaboration et al., A gravitational-wave standard siren measurement of the Hubble constant, Nature (London) 551, 85 (2017).

[50] B.F. Schutz, Determining the Hubble constant from gravitational wave observations, Nature (London) 323, 310 (1986).

[51] S. Fairhurst, Triangulation of gravitational wave sources with a network of detectors, New J. Phys. 11, 123006 (2009).

[52] B. P. Abbott et al. (LIGO Scientific and Virgo Collaboration), GW170104: Observation of a 50-Solar-Mass Binary Black Hole Coalescence at Redshift 0.2, Phys. Rev. Lett. 118, 221101 (2017).

[53] B. P. Abbott et al., GW170608: Observation of a 19 solarmass binary black hole coalescence, Astrophys. J. Lett. 851, L35 (2017).

[54] A. Rau et al., Exploring the optical transient sky with the Palomar transient factory, Publ. Astron. Soc. Pac. 121, 1334 (2009).

[55] Pan-STARRS, http://pswww.ifa.hawaii.edu/pswww/.

[56] Sky Mapper, http://skymapper.anu.edu.au.

[57] B. P. Abbott et al. LIGO Scientific Collaboration, Virgo Collaboration, GW170814: A Three-Detector Observation of Gravitational Waves from a Binary Black Hole Coalescence, Phys. Rev. Lett. 119, 141101 (2017).
[58] S. Fairhurst, Improved source localization with LIGOIndia, J. Phys. Conf. Ser. 484, 012007 (2014).

[59] J. Veitch, I. Mandel, B. Aylott, B. Farr, V. Raymond, C. Rodriguez, M. van der Sluys, V. Kalogera, and A. Vecchio, Estimating parameters of coalescing compact binaries with proposed advanced detector networks, Phys. Rev. D 85 104045 (2012).

[60] S. Vitale and M. Zanolin, Application of asymptotic expansions for maximum likelihood estimators' errors to gravitational waves from inspiraling binary systems: The network case, Phys. Rev. D 84, 104020 (2011).

[61] B. P. Abbott et al., The lsc-virgo white paper on instrument science (2016-2017 edition), Technical Report No. T1400226, LIGO and Virgo Scientific Collaborations, LIGO Internal Document No. T1400226, 2016.

[62] M. Punturo et al., The Einstein Telescope: A thirdgeneration gravitational wave observatory, Classical Quantum Gravity 27, 194002 (2010).

[63] M. Punturo et al., The third generation of gravitational wave observatories and their science reach, Classical Quantum Gravity 27, 084007 (2010).

[64] M. Punturo and H. Luck, Toward a third generation of gravitational wave observatories, Gen. Relativ. Gravit. 43, 363 (2011).

[65] B.P. Abbott et al., Exploring the sensitivity of next generation gravitational wave detectors, Classical Quantum Gravity 34, 044001.(2016).

[66] S. Vitale and M. Evans, Parameter estimation for binary black holes with networks of third generation gravitational-wave detectors, Phys. Rev. D 95, 064052 (2017).

[67] B. S. Sathyaprakash, B. Schutz, and C. Van Den Broeck, Cosmography with the Einstein Telescope, Classical Quantum Gravity 27, 215006 (2010).

[68] T. Regimbau, K. Siellez, D. Meacher, B. Gendre, and M. Boër, Revisiting coincidence rate between gravitational wave detection and short gamma-ray burst for the advanced and third generation, Astrophys. J. 799, 69 (2015).

[69] T. Regimbau et al., Mock data challenge for the Einstein Gravitational-Wave Telescope, Phys. Rev. D 86, 122001 (2012).

[70] D. Meacher, K. Cannon, C. Hanna, T. Regimbau, and B. S. Sathyaprakash, Second Einstein Telescope mock data and science challenge: Low frequency binary neutron star data analysis, Phys. Rev. D 93, 024018 (2016).

[71] X. Fan and M. Hendry, Multimessenger astronomy, arXiv:1509.06022.

[72] E. S. Phinney, Finding and using electromagnetic counterparts of gravitational wave sources, arXiv:0903.0098.

[73] T. Regimbau, D. Meacher, and M. Coughlin, Second Einstein Telescope mock science challenge: Detection of the gravitational-wave stochastic background from compact binary coalescences, Phys. Rev. D 89, 084046 (2014).

[74] M. L. Chan, C. Messenger, I. S. Heng, and M. Hendry, Binary neutron star mergers and third generation detectors: Localization and early warning, arXiv:1803.09680.

[75] W. Zhao and L. Wen, Localization accuracy of compact binary coalescences detected by the third-generation gravitational-wave detectors and implication for cosmology, Phys. Rev. D 97, 064031 (2018). 
[76] P. Raffai, L. Gondán, I. S. Heng, N. Kelecsényi, J. Logue, Z. Márka, and S. Márka, Optimal networks of future gravitational-wave telescopes, Classical Quantum Gravity 30, 155004 (2013).

[77] D. G. Blair et al., The science benefits and preliminary design of the southern hemisphere gravitational wave detector AIGO, J. Phys. Conf. Ser. 122, 012001 (2008).

[78] Y.-M. Hu, P. Raffai, L. Gondán, I. S. Heng, N. Kelecsényi, M. Hendry, Z. Márka, and S. Márka, Global optimization for future gravitational wave detectors' sites, Classical Quantum Gravity 32, 105010 (2015).

[79] F. Cavalier, M. Barsuglia, M.-A. Bizouard, V. Brisson, A.-C. Clapson, M. Davier, P. Hello, S. Kreckelbergh, N. Leroy, and M. Varvella, Reconstruction of source location in a network of gravitational wave interferometric detectors, Phys. Rev. D 74, 082004(2006).

[80] A. Pai, S. Dhurandhar, and S. Bose, A data-analysis strategy for detecting gravitational-wave signals from inspiraling compact binaries with a network of laserinterferometric detectors, Phys. Rev. D 64, 042004 (2001).

[81] J. Sylvestre, Position estimation from a network of interferometers, Classical Quantum Gravity 21, S775 (2004).

[82] S. Klimenko, G. Vedovato, M. Drago, G. Mazzolo, G. Mitselmakher, C. Pankow, G. Prodi, V. Re, F. Salemi, and I. Yakushin, Localization of gravitational wave sources with networks of advanced detectors, Phys. Rev. D 83, 102001 (2011).

[83] B. F. Schutz, Networks of gravitational wave detectors and three figures of merit, Classical Quantum Gravity 28, 125023 (2011).

[84] L. Wen and Y. Chen, Geometrical expression for the angular resolution of a network of gravitational-wave detectors, Phys. Rev. D 81, 082001 (2010).

[85] S. Fairhurst, Source localization with an advanced gravitational wave detector network, Classical Quantum Gravity 28, 105021 (2011).

[86] L. P. Singer et al., The first two years of electromagnetic follow-up with Advanced LIGO and Virgo, Astrophys. J. 795, 105 (2014).

[87] B. S. Sathyaprakash et al., LIGO-T1000251-v1: Report of the committee to compare the scientific cases for AHLV and HHLV, Technical Report LIGO-T1000251-v1, https:// dcc.ligo.org/LIGO-T1000251/public, 2010.

[88] B Iyer et al., LIGO-India: proposal of the consortium for Indian initiative in gravitational-wave observations (IndIGO), LIGO-India Technical Report LIGO-T1000251v1, https://dcc.ligo.org/LIGO-T1000251/public, 2013.

[89] C. Röver, R. Meyer, and N. Christensen, Coherent Bayesian inference on compact binary inspirals using a network of interferometric gravitational wave detectors, Phys. Rev. D 75, 062004 (2007).

[90] M. van der Sluys, I. Mandel, V. Raymond, V. Kalogera, C. Röver, and N. Christensen, Parameter estimation for signals from compact binary inspirals injected into LIGO data, Classical Quantum Gravity 26, 204010 (2009).

[91] V. Raymond, M. V. van der Sluys, I. Mandel, V. Kalogera, C. Röver, and N. Christensen, Degeneracies in sky localization determination from a spinning coalescing binary through gravitational wave observations: A Markov-chain
Monte Carlo analysis for two detectors, Classical Quantum Gravity 26, 114007 (2009).

[92] S. Nissanke, J. Sievers, N. Dalal, and D. Holz, Localizing compact binary inspirals on the sky using ground-based gravitational wave interferometers, Astrophys. J. 739, 99 (2011).

[93] J. Veitch et al., Parameter estimation for compact binaries with ground-based gravitational-wave observations using the LALInference software library, Phys. Rev. D 91, 042003 (2015).

[94] C. P. L. Berry et al., Parameter estimation for binary neutron-star coalescences with realistic noise during the Advanced LIGO era, Astrophys. J. 804, 114 (2015).

[95] C. L. Rodriguez, B. Farr, V. Raymond, W. M. Farr, T. B. Littenberg, D. Fazi, and V. Kalogera, Basic parameter estimation of binary neutron star systems by the Advanced LIGO/Virgo Network, Astrophys. J. 784, 119 (2014).

[96] L. P. Singer and L. R. Price, Rapid Bayesian position reconstruction for gravitational-wave transients, Phys. Rev. D 93, 024013 (2016).

[97] K. Grover, S. Fairhurst, B. F. Farr, I. Mandel, C. Rodriguez, T. Sidery, and A. Vecchio, Comparison of gravitational wave detector network sky localization approximations, Phys. Rev. D 89, 042004(2014).

[98] S. Fairhurst, Localization of transient gravitational wave sources: Beyond triangulation, Classical Quantum Gravity 35, 105002 (2018).

[99] LIGO Scientific Collaboration, Advanced ligo, Classical Quantum Gravity 32, 7 (2014).

[100] M. Abernathy et al., ET design study, http://www.et-gw .eu/index.php/etdsdocument, 2011.

[101] J. Aasi et al., Enhanced sensitivity of the ligo gravitational wave detector by using squeezed states of light, Nat. Photonics 7, 613 (2013).

[102] D. Madden-Fong, B. Shapiro and B. Lantz, LIGO III quad pendulum conceptual design optimization, Technical Report, LIGO and Virgo Scientific Collaborations, Technical Report No. LIGO-T1300786-v6, https://dcc.ligo.org/ LIGO-T1300786/public, 2014.

[103] B. Shapiro et al., Cryogenically cooled ultra low vibration silicon mirrors for gw observatories, Technical Report No. P1600301, LIGO and Virgo Scientific Collaborations, https://dcc.ligo.org/LIGO-P1600301/public, 2016.

[104] J. Westland and A. Heptonstall, Finite element analysis of the third generation mirror suspension systems for voyager, Technical Report, LIGO and Virgo Scientific Collaborations, Technical Report No. LIGO-T1600293-v4, https://dcc.ligo.org/LIGO-T1600293/public, 2016.

[105] T. Hinderer, B. D. Lackey, R. N. Lang, and J. S. Read, Tidal deformability of neutron stars with realistic equations of state and their gravitational wave signatures in binary inspiral, Phys. Rev. D 81, 123016 (2010).

[106] B. S. Sathyaprakash et al., Scientific objectives of Einstein Telescope, Classical Quantum Gravity 29, 124013 (2012).

[107] R.-G. Cai and T. Yang, Estimating cosmological parameters by the simulated data of gravitational waves from the Einstein Telescope, Phys. Rev. D 95, 044024 (2017).

[108] A. Freise, S. Chelkowski, S. Hild, W. Del Pozzo, A. Perreca, and A. Vecchio, Triple Michelson interferometer 
for a third-generation gravitational wave detector, Classical Quantum Gravity 26, 085012 (2009).

[109] K. S. Thorne, in Three Hundred Years of Gravitation, edited by S.W. Hawking and W. Israel (Cambridge University Press, Cambridge, 1987), Chap. 9, p. 330.

[110] R. Essick, S. Vitale, and M. Evans, Frequency-dependent responses in third generation gravitational-wave detectors, Phys. Rev. D 96, 084004 (2017).

[111] B. Allen, W. G. Anderson, P. R. Brady, D. A. Brown, and J. D. E. Creighton, FINDCHIRP: An algorithm for detection of gravitational waves from inspiraling compact binaries, Phys. Rev. D 85, 122006 (2012).

[112] I. Harry and S. Fairhurst, A targeted coherent search for gravitational waves from compact binary coalescences, Phys. Rev. D 83, 084002 (2011).

[113] S. Klimenko, S. Mohanty, M. Rakhmanov, and G. Mitselmakher, Constraint likelihood analysis for a network of gravitational wave detectors, Phys. Rev. D 72, 122002 (2005).

[114] D. M. Macleod, I. W. Harry, and S. Fairhurst, A fullycoherent all-sky search for gravitational-waves from compact binary coalescences, Phys. Rev. D 93, 064004 (2016).

[115] B. P. Abbott et al., GW150914: First results from the search for binary black hole coalescence with Advanced LIGO, Phys. Rev. D 93, 122003 (2016).

[116] A. H. Nitz, T. Dent, T. D. Canton, S. Fairhurst, and D. A. Brown, Detecting binary compact-object mergers with gravitational waves: Understanding and Improving the sensitivity of the PyCBC search, Astrophys. J. 849, 118 (2017).

[117] A. R. Williamson, C. Biwer, S. Fairhurst, I. W. Harry, E. Macdonald, D. Macleod, and V. Predoi, Improved methods for detecting gravitational waves associated with short gamma-ray bursts, Phys. Rev. D 90, 122004 (2014).

[118] F. Özel, D. Psaltis, R. Narayan, and A. S. Villarreal, On the mass distribution and birth masses of neutron stars, Astrophys. J. 757, 55 (2012).

[119] O. Pejcha, T. A. Thompson, and C.S. Kochanek, The observed neutron star mass distribution as a probe of the supernova explosion mechanism, Mon. Not. R. Astron. Soc. 424, 1570 (2012).

[120] T. Totani, Cosmological gamma-ray bursts and evolution of galaxies, Astrophys. J. Lett. 486, L71 (1997).

[121] P. A. R. Ade et al. (Planck Collaboration), Planck 2015 results. XIII. Cosmological parameters, Astron. Astrophys. 594, A13 (2016).

[122] P. Madau and M. Dickinson, Cosmic star-formation history, Annu. Rev. Astron. Astrophys. 52, 415 (2014).

[123] K. Belczynski, R. Perna, T. Bulik, V. Kalogera, N. Ivanova, and D. Q. Lamb, A study of compact object mergers as short gamma-ray burst progenitors, Astrophys. J. 648, 1110 (2006).

[124] D. Guetta and T. Piran, The BATSE-Swift luminosity and redshift distributions of short-duration GRBs, Astronomy Astrophysics 453, 823 (2006).
[125] S. R. Taylor and J. R. Gair, Cosmology with the lights off: standard sirens in the Einstein Telescope era, Phys. Rev. D 86, 023502 (2012).

[126] J. Clark, H. Evans, S. Fairhurst, I. W. Harry, E. Macdonald, D. Macleod, P. J. Sutton, and A. R. Williamson, Prospects for joint gravitational wave and short gamma-ray burst observations, Astrophys. J. 809, 53 (2015).

[127] D. Lazzati, D. López-Cámara, M. Cantiello, B. J. Morsony, R. Perna, and J.C. Workman, Off-axis prompt x-ray transients from the cocoon of short gamma-ray bursts, Astrophys. J. Lett. 848, L6 (2017).

[128] D. Lazzati et al., Late time afterglow observations reveal a collimated relativistic jet in the ejecta of the binary neutron star merger GW170817, arXiv:1712.03237.

[129] C. J. Fontes et al., A line-smeared treatment of opacities for the spectra and light curves from macronovae, arXiv:1702.02990.

[130] J. Barnes, D. Kasen, M.-R. Wu, and G. Martínez-Pinedo, Radioactivity and thermalization in the ejecta of compact object mergers and their impact on kilonova light curves, Astrophys. J. 829, 110 (2016).

[131] B. D. Metzger, Kilonovae, Living Rev. Relativity 20, 3 (2017).

[132] V. A. Villar et al., The combined ultraviolet, optical, and near-infrared light curves of the kilonova associated with the binary neutron star merger GW170817: Unified data set, analytic models, and physical implications, Astrophys. J. 851, L21 (2017).

[133] N. R. Tanvir, A. J. Levan, A. S. Fruchter, J. Hjorth, R. A. Hounsell, K. Wiersema, and R. L. Tunnicliffe, A "kilonova" associated with short-duration gamma-ray burst 130603B, Nature (London) 500, 547 (2013).

[134] E. Berger, W. Fong, and R. Chornock, An r-process Kilonova Associated with the short-hard GRB 130603B, Astrophys. J. 774, L23 (2013).

[135] J. A. Tyson and the LSST Collaboration, Large synoptic survey telescope: Overview, arXiv:astro-ph/0302102.

[136] T. D. Canton, Efficient searches for spinning compact binaries with advanced gravitational-wave observatories, Ph.D. thesis, Leibniz University Hannover, Hannover, Germany, 2015.

[137] B. P. Abbott et al., Calibration of the Advanced LIGO detectors for the discovery of the binary black-hole merger GW150914, Phys. Rev. D 95, 062003 (2017).

[138] W. D. Pozzo, Inference of the cosmological parameters from gravitational waves: application to second generation interferometers, Phys. Rev. D 86, 043011 (2012).

[139] C. Messenger and J. Read, Measuring a Cosmological Distance-Redshift Relationship Using Only Gravitational Wave Observations of Binary Neutron Star Coalescences, Phys. Rev. Lett. 108, 091101 (2012). 